\title{
Dielectric properties of perovskite crystals
}

\author{
B S SEMWAL and N S PANWAR \\ Physics Department, Garhwal University, Srinagar 246 174, India
}

\begin{abstract}
The soft mode dynamical model has been used to study the dielectric properties of Perowskite-type crystals. The model Hamiltonian proposed by Pytte has been modified and designed in terms of creation and annihilation operators. The correlations appearing in the dynamical equation have been evaluated using double time thermal retarded Green's function and Dyson's equation. Without any decoupling the higher order correlations have been evaluated using the renormalized Hamiltonian and thus, all possible interactions among phonons have been taken into account. The expressions for phonon frequencies and widths have been _calculated. Using appropriate parameters the softening of different modes at different transition temperatures give rise lo a series of transitions from cubic to tetregonal, orthorhombic or trigonal phases. The significantly temperature-dependent modes are considered responsible for damping constant, dielectrie constant, tangent loss and attenuation constant for these crystals. The dielectric properties are directly related to the optical phonon frequencies and widths and acoustic attenuation to the acoustic and optical phonon widths. Using suitable approximations, the model explains the experimental results on dielectric properties and acoustic attentation reported for $\mathrm{LiNbO}_{3}, \mathrm{SrTiO}_{3}, \mathrm{BaTiO}_{3}$ and $\mathrm{LaAlO}_{3}$.
\end{abstract}

Keywords. Soft mode; frequency shift and width; dielectric constant; loss tangent; acoustic attenuation.

\section{Introduction}

The oscillations of atoms in a solid are responsible for different characteristics such as specific heat, optical, dielectric and electrical properties. The anharmonicity in solids is responsible for the existence of thermal expansion, temperature dependence of elastic constants, lattice thermal conductivity, variation of specific heat at different temperatures, etc. Many attempts have been made theoretically and experimentally to explain these phenomena in terms of anharmonicity. Extensive reviews (Sernwal 1972; Semwal and Sharma 1972, 1974) are available discussing the contribution of antharmonicity in various properties of crystals.

In displacive dielectrics, the transitions are associated with the displacement of a whole sublattice of ions of one type relative to another sublattice. The atonic displacements at the transition point are small compared to the unit cell dimensions. In displacive compounds the phonon coordinate is the order parameter and the critical fluctuations of the ordering quantity are carried by the soft phonons- the phonon modes which are significantly temperature-dependent and cease at the transition temperature. In the other types of transitions, which are called orderdisorder type, as $\mathrm{KH}_{2} \mathrm{PO}_{4}$, the transition is associated with the tunnelling of proton through a barrier between two positions of minimum potential energy in the double well potential in the hydrogen bond at the transition temperature (Kobayashi 1968). The displacive-type crystals with $\mathrm{ABO}_{3}$ structure (where $\mathrm{A}$ is a first, second, fourth or even fifth group of ions of appropriate valency and $B$ is a transitional metal ion such as $\mathrm{Ti}, \mathrm{Nb}, \mathrm{Ta}, \mathrm{Zr}$ ) in which the transition occurs due to the rotation of $\mathrm{BO}_{6}$ octahedra are known as perovskite type, e.g. $\mathrm{SrTiO}_{3}, \mathrm{PrAlO}_{3}, \mathrm{KMnF}_{3}, \mathrm{LaAlO}_{3}$, $\mathrm{BaTiO}_{3}, \mathrm{PbTiO}_{3}$ and various solid solutions. These transitions are associated with 
a phonon instability (Cochran 1959; Anderson 1960) more specifically with the condensation of different sets of modes at the Brillouin zone centre (at $q=0$ ) in the case of ferroelectrics e.g. $\mathrm{BaTiO}_{3}$ or at the zone boundary (at $q=(1 / 2,1 / 2,1 / 2)$ ) in the case of antiferroelectrics e.g. $\mathrm{SrTiO}_{3}$. One may use a set of three basic modes corresponding to rotations of $\mathrm{BO}_{6}$ octahedra around the three cube axes. In $\mathrm{SrTiO}_{3}$, one of the triply degenerate modes condenses (Cowley et al 1969) and in $\mathrm{LaAlO}_{3}$ a linear combination of all three modes (Cochran and Zia 1968). The rotation of $\mathrm{BO}_{6}$ octahedra about different cube axes give different structures in the distorted phase. For example, in $\mathrm{SrTiO}_{3}$, the cubic high temperature structure undergoes a retragonal distortion at the transition temperature $\left(106^{\circ} \mathrm{K}\right)$ corresponding to the rotation of $\mathrm{TiO}_{6}$ octahedra about a cube axis. In $\mathrm{LaAlO}_{3}$ it undergoes a trigonal distortion, described by the rotation of oxygen octahedra about the cube diagonal. In some materials a series of transitions from high- to low-symmetry structures are observed, e.g. $\mathrm{BaTiO}_{3}$ undergoes a ferroelectric transition at $121^{\circ} \mathrm{C}$ from cubic to tetragonal structure and goes further to the orthorhombic $\left(5^{\circ} \mathrm{C}\right)$ and trigonal structure $\left(-80^{\circ} \mathrm{C}\right)$,

It is revealed experimentally and theoretically that the soft mode plays a fundamental role in perovskite crystals because the dielectric constant, loss tangent, acoustic attenuation and Curie temperature depend explicitly on the soft mode frequency.

In the present study, the model Hamiltonian proposed by Pytte (1972) and designed in terms of creation and annihilation operators was modificd by considering all possible interactions among different vibrational modes. The correlation functions were evaluated using the Green's function technique and Dyson's equation. The higher order correlations appearing in the dynamical equation were not decoupled but evaluated using a renormalized Hamiltonian, expressions for phonon frequencies and widths and hence the dielectric constant, loss tangent and acoustic attenuation were obtained. Pytte (1972) decoupled the correlations in the very beginning and could not obtain the frequency shifts and widths and hence the related properties.

\section{The model Hamiltonian and Green's function}

The model Hamiltonian in the operator form is obtained as

where

$$
\begin{aligned}
H= & \frac{1}{4} \sum_{\dot{j}_{1} q_{1}} \Omega_{\lambda_{1}}\left(q_{1}\right) B_{\lambda_{1}}^{\dagger}\left(q_{1}\right) B_{\lambda_{1}}\left(q_{1}\right)+\frac{1}{4} \sum_{\mu q_{1}} \omega_{j}\left(q_{1}\right) B_{\mu}^{\dagger}\left(q_{1}\right) B_{\mu}\left(q_{1}\right) \\
& +\sum \alpha S_{\mu}^{\dagger}\left(q_{1}\right) S_{\mu}\left(q_{1}\right)+\sum \beta S_{\lambda_{1}}^{\dagger}\left(q_{1}\right) S_{\lambda_{1}}\left(q_{1}\right)+\sum \eta S_{\mu}^{\dagger}\left(q_{1}\right) S_{\lambda_{1}}\left(q_{1}\right) \\
& +\sum \delta S_{\lambda_{2}}\left(q_{2}\right) S_{\lambda_{1}}^{\dagger}\left(q_{1}\right) S_{\lambda_{1}}\left(q_{1}\right)+\sum \phi S_{\mu}\left(q_{2}\right) S_{\lambda_{1}}^{\dagger}\left(q_{1}\right) S_{\lambda_{1}}\left(q_{1}\right) \\
& +\sum \theta S_{\mu}\left(q_{2}\right) S_{\lambda_{1}}\left(q_{1}\right)+\sum \psi S_{\lambda_{1}}^{\dagger}\left(q_{1}\right) S_{\lambda_{1}}\left(q_{1}\right) S_{\lambda_{2}}^{\dagger}\left(q_{2}\right) S_{\lambda_{2}}\left(q_{2}\right) \\
& +\sum \eta S_{\mu}^{\dagger}\left(q_{2}\right) S_{\mu}\left(q_{2}\right) S_{\lambda_{1}}^{\dagger}\left(q_{1}\right) S_{\lambda_{1}}\left(q_{1}\right)
\end{aligned}
$$

$$
\begin{aligned}
& \sum \alpha=\frac{1}{4} \sum_{\mu q_{1}} \frac{\omega_{0}^{2}\left(\mu q_{1}\right)}{\omega_{\mu}\left(q_{1}\right)}+\frac{1}{4} \sum_{j j, \mu q_{1}} g_{i j \lambda} A_{\lambda}^{2} \frac{\alpha_{i}^{\dagger}\left(\mu q_{1}\right) \alpha_{j}\left(\mu q_{1}\right)}{\omega_{\mu}\left(q_{1}\right)}, \\
& \sum \beta=\left[\sum_{i 1 q_{1}}\left(\frac{1}{4} \Omega_{0}^{2}+\frac{3}{4} \Gamma_{1} \sum_{\lambda} A_{\lambda}^{2}+\frac{\Gamma_{2}}{2} \sum_{\lambda \neq \lambda^{\prime}} A_{\lambda^{\prime}}^{2}+\frac{1}{4} \sum_{i, \lambda} g_{i j \lambda} e_{i} e_{j}\right)\right.
\end{aligned}
$$




$$
\begin{aligned}
& \times b_{\dot{\lambda \lambda_{1}}}^{\dagger}\left(q_{1}\right) b_{\lambda \dot{\lambda}_{1}}\left(q_{1}\right) / \Omega_{\lambda_{1}}\left(q_{1}\right)+\sum_{i \lambda^{2} \lambda_{1} q_{1}} G_{i \lambda^{\prime}} e_{i} \frac{b_{\lambda \lambda_{1}}^{\dagger}\left(q_{1}\right) b_{\lambda \lambda_{1}}\left(q_{1}\right)}{2 \Omega_{\lambda_{1}}\left(q_{1}\right)}
\end{aligned}
$$

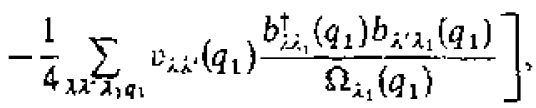

$$
\begin{aligned}
& \sum \gamma=\left[\sum_{\mu \lambda_{1} q_{1}}\left(\sum_{i \lambda \lambda^{\prime}} G_{i \lambda \lambda^{\prime}} A_{\lambda^{*}}+\sum_{i j \lambda} g_{i j \lambda^{\prime}} A_{\lambda^{\prime}} e_{j}\right) \alpha_{1}^{\dagger}\left(j \alpha q_{1}\right) b_{\lambda \lambda_{1}}\left(q_{1}\right) /\left(o_{\mu}\left(q_{1}\right) \Omega_{\lambda_{1}}\left(q_{1}\right)\right)^{1 / 2}\right] \text {, } \\
& \sum \phi=\left[\sum _ { \mu , \lambda _ { 1 } q _ { 1 q _ { 2 } } } \left(\sum_{i, \lambda^{\prime}} G_{i \lambda^{\prime}} \alpha_{i}\left(\mu q_{2}\right) b_{\lambda_{\lambda}}^{\dagger}\left(q_{1}\right) b_{\lambda_{\lambda_{1}}}\left(q_{1}\right)\right.\right.
\end{aligned}
$$

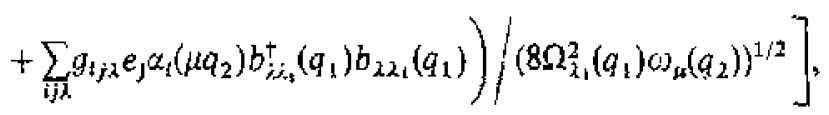

$$
\begin{aligned}
& \sum \delta=\left[\sum _ { \lambda _ { 1 } \lambda _ { 2 q _ { 1 } q _ { 2 } } } \left(\Gamma_{2} \sum_{\lambda \neq \lambda_{\lambda^{\prime}}} \frac{A_{\lambda} b_{\lambda_{\lambda \lambda_{2}}}\left(q_{2}\right) b_{\lambda_{1}}^{\dagger}\left(q_{1}\right) b_{\lambda \lambda_{1}}\left(q_{1}\right)}{\left(2 \Omega_{\lambda_{1}}^{2}\left(q_{1}\right) \Omega_{\lambda_{2}}\left(q_{2}\right)\right)^{1 / 2}}\right.\right. \\
& \left.+\frac{\Gamma_{1}}{2} \sum_{\lambda} \frac{A_{2} b_{\lambda \lambda_{2}}\left(a_{2}\right) b_{\lambda_{1}}^{\dagger}\left(q_{1}\right) b_{\lambda_{1}}\left(a_{1}\right)}{\left(2 \Omega_{\lambda_{1}}^{2}\left(q_{1}\right) \Omega_{\lambda_{2}}\left(q_{2}\right)\right)^{1 / 2}}\right] \text {. }
\end{aligned}
$$

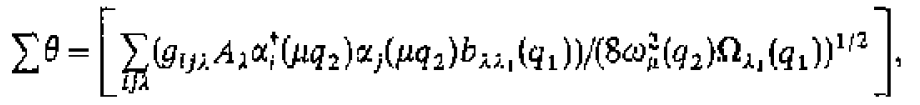

$$
\begin{aligned}
& \sum \psi=\left[\sum _ { \lambda _ { 1 } \lambda _ { \lambda q _ { 1 } q _ { 2 } } } \left(\frac{\Gamma_{1}}{2} \sum_{\lambda} b_{\lambda_{1}}^{\dagger}\left(a_{1}\right) b_{\lambda_{\lambda_{2}}}\left(q_{2}\right) b_{\lambda_{1}}^{\dagger}\left(q_{1}\right) b_{\lambda_{\lambda_{1}}}\left(q_{1}\right)\right.\right. \\
& \left.\left.+\frac{\Gamma_{1}}{2} \sum_{\lambda \neq \lambda^{\prime}} b_{\lambda_{1}}^{\dagger}\left(q_{2}\right) b_{\lambda \lambda_{2}}\left(q_{2}\right) b_{\lambda_{1}}^{\dagger}\left(q_{1}\right) b_{\lambda^{\prime} \lambda_{1}}\left(q_{1}\right)\right) /\left(4 \Omega_{\lambda_{1}}\left(q_{1}\right) \Omega_{\lambda_{2}}\left(q_{2}\right)\right)\right] \\
& \sum \eta=\left[\sum_{i j \lambda} g_{i j \lambda} \alpha_{i}\left(\mu q_{1}\right) \alpha_{j}\left(\mu q_{1}\right) b_{j \lambda_{1}}^{\dagger}\left(q_{1}\right) b_{\lambda_{1}}\left(q_{1}\right) /\left(8 \omega_{\mu}\left(q_{1}\right) \Omega_{\lambda_{1}}\left(q_{1}\right)\right)\right] \\
& S_{\lambda}(q)=\left[a_{\lambda}(q)+a_{\lambda}^{\dagger}(-q)\right], \quad B_{\lambda}(q)=\left[a_{\lambda}(q)-a_{\lambda}^{\dagger}(-q)\right]
\end{aligned}
$$

where the symbols are the same as used by Pytte (1972).

The correlations appearing in the phonon response function can be evaluated using the double time thermal retarded Green's function (Zubarev 1960)

$$
\begin{aligned}
G\left(t-t^{\prime}\right) & =\left\langle S_{\lambda_{3}}\left(q_{3}\right)_{t}: S_{\lambda_{4}}^{t}\left(q_{4}\right)_{t^{r}}\right\rangle \\
& =-j \theta\left(t-t^{\prime}\right)\left\langle\left[S_{\lambda_{3}}\left(q_{3}\right)_{1}: S_{\lambda_{4}}^{\dagger}\left(q_{4}\right)_{t^{\prime}}\right]\right\rangle,
\end{aligned}
$$

where the angular brackets denote the average over the large canonical ensemble and $\theta(t)$ is the Heaviside step fonction having properties

$$
\theta(t)=1 \text { for } t>0 \text { and } 0 \text { for } t<0, j=(-1)^{1 / 2} \text {. }
$$

Differentiating (3) twice with respect to $t$, using the Hamiltonian (1) and taking Fourier transformation, one obtains

$$
\left(\omega^{2}-\widetilde{\Omega}_{\lambda_{3}}^{2}\left(q_{3}\right)\right) G(\omega)=\frac{\Omega_{\lambda_{3}}\left(q_{3}\right)}{\pi} \frac{\delta_{\lambda_{3} \lambda_{4}} \delta_{43 q_{4}}}{\pi}+\frac{\Omega_{\lambda_{3}}\left(q_{3}\right)}{\pi} \mathrm{F}(\omega),
$$


where

$$
\begin{aligned}
& \tilde{\Omega}_{\lambda_{3}}^{2}\left(q_{3}\right)=\left\{\left(\Omega_{0}^{2}+3 \Gamma_{1} \sum_{\lambda} A_{\lambda}^{2}+2 \Gamma_{2} \sum_{\lambda \neq \lambda^{\prime}} A_{\lambda^{\prime}}^{2}+\sum_{i j \lambda} g_{j \lambda \lambda} e_{l} e_{J}\right) b_{\lambda_{\lambda}}^{\dagger}\left(q_{3}\right) b_{\lambda \lambda_{3}}\left(q_{3}\right)\right.
\end{aligned}
$$

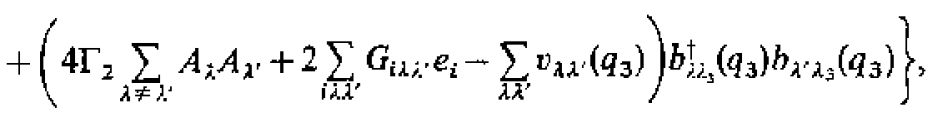

and the higher order Green's functions

with

$$
\Gamma\left(t-t^{\prime}\right)=\left\langle f_{\lambda_{3}}\left(q_{3}\right)_{t}: S_{\lambda_{4}}^{\dagger}\left(q_{4}\right)_{t^{\prime}}\right\rangle
$$

$$
\begin{aligned}
f_{\lambda_{3}}\left(q_{3}\right)= & \pi\left[{v_{1}}_{1} S_{\mu}\left(q_{3}\right)+\bar{\delta}_{1} S_{\lambda_{1}}\left(q_{1}\right) S_{\lambda_{1}}\left(q_{1}\right)+\bar{\delta}_{2} S_{\lambda_{3}}^{\dagger}\left(q_{3}\right) S_{\lambda_{1}}\left(q_{1}\right)\right. \\
& +\phi_{1} S_{\mu}\left(q_{1}\right) S_{\lambda_{3}}\left(q_{3}\right)+\theta_{1} S_{\mu}^{\dagger}\left(q_{2}\right) S_{\mu}\left(q_{1}\right)+\psi_{1} S_{\lambda_{3}}\left(q_{3}\right) S_{\lambda_{1}}^{\dagger}\left(q_{1}\right) S_{\lambda_{1}}\left(q_{1}\right) \\
& \left.+\eta_{1} S_{\mu}\left(q_{1}\right) S_{\mu}\left(q_{1}\right) S_{\lambda_{3}}\left(q_{3}\right)\right] .
\end{aligned}
$$

Here, $\gamma_{1}, \delta_{1}, \theta_{1}$, etc. are the corresponding values of $\gamma, \delta_{3}, \theta$, etc. from (2) obtained by evaluating the respective commutations appearing in the Green's function (3).

$P(\omega)$ is calculated by differentiating (6) twice with respect to $t^{\prime}$ using Hamiltonian (1) and then taking Fourier transformation, one obtains

where

$$
\Gamma(\omega)=\frac{1}{\left[\omega^{2}-\tilde{\Omega}_{\lambda_{4}}^{2}\left(q_{4}\right)\right]}\left(F+\frac{\Omega_{\lambda_{4}}\left(q_{4}\right)}{\pi} \mathbb{F}(\omega)\right)
$$

and

$$
\begin{aligned}
& F=\left\{\left(\Gamma_{1} \sum_{\lambda}\left|b_{\lambda_{4}}^{\dagger}\left(q_{4}\right) b_{\lambda \lambda_{4}}\left(q_{4}\right)\right|^{2}+2 \Gamma_{2} \sum_{\lambda \neq \lambda^{\prime}}\left|b_{\lambda \lambda_{4}}^{\dagger}\left(q_{4}\right) b_{\lambda^{\prime} \lambda_{4}}\left(q_{4}\right)\right|^{2}\right)\right. \\
& \times \frac{\operatorname{coth}\left(\beta \tilde{\Omega}_{\lambda_{4}}\left(q_{4}\right) / 2\right)}{\tilde{\Omega}_{\lambda_{4}}\left(q_{4}\right)}+\left.\left(\frac{\Gamma_{1}}{2} \sum_{\lambda} \mid b\right)_{\lambda_{4}}^{\dagger}\left(q_{4}\right) b_{\lambda_{\lambda_{1}}}\left(q_{1}\right)\right|^{2} \\
& \left.+\Gamma_{2} \sum_{\lambda \neq \lambda^{*}}\left|b_{\lambda_{4}}^{\dagger}\left(q_{4}\right) b_{\lambda^{\prime} \lambda_{1}}\left(q_{1}\right)\right|^{2}\right) \frac{\operatorname{coth}\left(\beta \tilde{\Omega}_{\lambda_{1}}\left(q_{1}\right) / 2\right)}{\tilde{\Omega}_{\lambda_{1}}\left(q_{1}\right)} \\
& \left.+\left(\frac{1}{2} \sum_{i j \lambda} g_{i j \lambda} \alpha_{i}\left(\mu q_{1}\right) z_{j}\left(\mu q_{2}\right) b_{i \alpha_{4}}^{\dagger}\left(q_{4}\right) b_{\lambda \lambda_{4}}\left(q_{4}\right)\right) \frac{\operatorname{coth}\left(\beta \tilde{\omega}_{\mu}\left(q_{1}\right) / 2\right.}{\omega_{\mu}\left(q_{1}\right)}\right\},
\end{aligned}
$$

$$
\mathbb{F}\left(t-t^{\prime}\right)=\left\langle f_{\lambda_{3}}\left(q_{3}\right)_{t} f_{\rangle_{4}}^{\dagger}\left(q_{4}\right)_{t}\right\rangle
$$

Substituting the value of $F(\omega)$ from (8) in (4) and using Dyson's equation (Gairola and Semwal 1977) one obtains

$$
G(\omega)=\frac{\Omega_{\lambda_{4}}\left(q_{4}\right) \delta_{\lambda_{3} \lambda_{4}} \delta_{q_{34}}}{\pi\left[\omega^{2}-\bar{\Omega}_{\lambda_{4}}^{2}\left(q_{4}\right)-\frac{\Omega_{\lambda_{4}}\left(q_{4}\right)_{F}}{\pi} F(\omega)\right]},
$$

where the renormalized frequency is

$$
\tilde{\Omega}_{\lambda_{4}}^{2}\left(q_{4}\right)=\tilde{\Omega}_{\lambda_{4}}^{2}\left(q_{4}\right)+F .
$$

The higher order Green's functions $\mathbb{F}(\omega)$ are calculated using the renormalized 
Hamiltonian

$$
\begin{aligned}
H_{\mathrm{ren}}= & \frac{1}{4} \sum_{\mu, \lambda}\left(\frac{\Omega_{\lambda}^{2}(q)}{\Omega_{\lambda}(q)} S_{\lambda}^{\dagger}(q) S_{\lambda}(q)+\Omega_{\lambda}(q) B_{\lambda}^{\dagger}(q) B_{\lambda}(q)\right. \\
& \left.+\frac{\tilde{\omega}_{\mu}^{2}(q)}{\omega_{\mu}(q)} S_{\mu}^{\dagger}(q) S_{\mu}(q)+\omega_{\mu}(q) B_{\mu}^{\dagger}(q) B_{\mu}(q)\right),
\end{aligned}
$$

and (11) can be written as

where

$$
G(\omega)=\Omega_{\lambda_{4}}\left(q_{4}\right) / \pi\left[\omega^{2}-\Omega_{\lambda_{4}}^{2}\left(q_{4}\right)-p_{\lambda_{4}}\left(q_{4}, \omega\right)\right]
$$

and

$$
p_{\lambda_{4}}\left(q_{4}, \omega\right)=\sum_{i=1}^{7} G_{i}^{\lambda}\left(q_{4}, \omega\right)
$$

$$
\begin{aligned}
G_{2}^{\lambda}\left(q_{4}, \omega\right)= & \left\{\sum _ { q _ { 1 } } \left(\Gamma_{1} \sum_{\lambda} A_{\lambda} b_{\lambda_{1}}^{\dagger}\left(q_{1}\right) b_{j \lambda_{4}}^{\dagger}\left(q_{4}\right) b_{\lambda \lambda_{1}}\left(q_{1}\right)\right.\right. \\
& \left.+2 \Gamma_{2} \sum_{\lambda \neq \lambda_{\lambda}^{\prime}} A_{\lambda} b_{\lambda_{\lambda_{4}}}^{\dagger}\left(q_{4}\right) b_{\lambda_{\lambda_{1}}}^{\dagger}\left(q_{1}\right) b_{\lambda^{\prime} \lambda_{1}}\left(q_{1}\right)\right)^{2} \\
& \left.\times \frac{n_{\lambda_{1}}\left(q_{1}\right)}{\left[\omega^{2}-4 \hat{\Omega}_{\lambda_{1}}^{2}\left(q_{1}\right)\right] \tilde{\Omega}_{\lambda_{1}}\left(q_{1}\right)}\right\}, \\
G_{3}^{\lambda}\left(q_{4}, \omega\right)= & \left\{\sum _ { q _ { 1 } } \left(\Gamma_{1} \sum_{\lambda} A_{\lambda} b_{\lambda \lambda_{1}}\left(q_{1}\right) b_{\lambda_{\lambda_{4}}}^{\dagger}\left(q_{4}\right) b_{\lambda \lambda}\left(q_{4}\right)\right.\right.
\end{aligned}
$$

$$
\begin{aligned}
& \left.+2 \Gamma_{2} \sum_{\lambda \neq \lambda^{\prime}} A_{\dot{\lambda}} b_{i \lambda_{1}}^{+}\left(q_{1}\right) b_{\lambda / \lambda_{4}}\left(q_{4}\right) b_{\lambda^{\prime} / \lambda_{4}}\left(q_{4}\right)\right)^{2} \\
& \left.\times \frac{1}{\overline{\Omega_{i_{1}}\left(q_{1}\right) \bar{\Omega}_{\lambda_{4}}\left(q_{4}\right)}}\left[\sum \frac{\left(n_{\lambda_{4}}\left(q_{4}\right) \pm n_{\lambda_{1}}\left(q_{1}\right)\right)\left(\bar{\Omega}_{\lambda_{1}}\left(q_{1}\right) \pm \bar{\Omega}_{\lambda_{4}}\left(q_{4}\right)\right)}{\left[\omega^{2}-\left(\tilde{\Omega}_{\lambda_{1}}\left(q_{1}\right) \pm \bar{\Omega}_{\lambda_{4}}\left(q_{4}\right)\right)^{2}\right]}\right]\right\}
\end{aligned}
$$

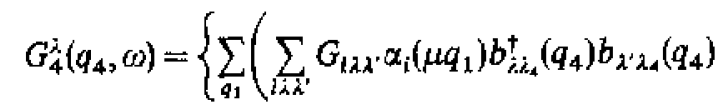

$$
\begin{aligned}
& \left.+\sum_{i j \lambda} g_{i j \lambda} e_{j} \alpha_{i}\left(\mu q_{1}\right) b_{i \lambda_{4}}^{\dagger}\left(q_{4}\right) b_{\lambda \lambda_{4}}\left(q_{4}\right)\right)^{2} \frac{1}{\hat{\omega}_{\mu}\left(q_{1}\right) \hat{\Omega}_{\lambda_{4}}\left(q_{4}\right)} \\
& \left.\times\left[\sum_{ \pm} \frac{\left(n_{\lambda_{4}}\left(q_{4}\right) \pm n_{\mu}\left(q_{1}\right)\right)\left(\tilde{\omega}_{\mu}\left(q_{1}\right) \pm \tilde{\Omega}_{\lambda_{1}}\left(q_{4}\right)\right)}{\left[\omega^{2}-\left(\tilde{\omega}_{\mu}\left(q_{1}\right) \pm \tilde{\Omega}_{\lambda_{4}}\left(q_{4}\right)\right)^{2}\right]}\right]\right\}, \\
& G_{5}^{\lambda}\left(q_{4}, \omega\right)=\left\{\sum_{q_{1}} 2\left(\sum_{i j \lambda} g_{i j \lambda} A_{\lambda} b_{i \lambda_{4}}\left(q_{4}\right) \alpha_{i}^{\dagger}\left(\mu q_{1}\right) \alpha_{j}\left(\mu q_{1}\right)\right)^{2}\right. \\
& \left.\times \frac{n_{\mu}\left(q_{1}\right)}{\overline{\omega_{\mu}}\left(q_{1}\right)\left(\omega^{2}-4 \tilde{\omega}_{\mu}^{2}\left(q_{1}\right)\right)}\right\} \text {. }
\end{aligned}
$$




$$
\begin{aligned}
& G_{\tilde{6}}^{\lambda}\left(q_{4}, \omega\right)=\left\{\sum_{q_{1}} \frac{3}{4}\left(\frac{\Gamma_{1}}{2} \sum_{\lambda}\left|b_{\lambda \lambda_{4}}\left(q_{4}\right) b_{\lambda \lambda_{1}}\left(q_{1}\right)\right|^{2}+\Gamma_{2} \sum_{\lambda \neq \lambda^{\prime}}\left|b_{\lambda_{4}}^{+}\left(q_{4}\right) b_{\lambda^{\prime} \lambda_{1}}\left(q_{1}\right)\right|^{2}\right)^{2} \mid\right. \\
& \widehat{\Omega}_{\lambda_{3}}^{2}\left(q_{1}\right) \Omega_{\lambda_{4}}\left(q_{4}\right)\left[\sum \frac{\left(1 \pm 2 n_{\lambda_{1}}\left(q_{1}\right) n_{\lambda_{1}}\left(q_{4}\right)+n_{\lambda_{1}}^{2}\left(q_{1}\right)\right)\left(\tilde{\Omega}_{\lambda_{4}}\left(q_{4}\right) \pm 2 \bar{\Omega}_{\lambda_{1}}^{*}\left(q_{2}\right)\right)}{\left[\omega^{2}-\left(\Omega_{\lambda_{4}}\left(q_{4}\right) \pm 2 \Omega_{\lambda_{1}}\left(q_{4}\right)\right)^{2}\right]}\right. \\
& \left.\left.+\frac{2 \tilde{\Omega}_{\lambda_{4}}\left(q_{4}\right)\left(1+2 n_{\lambda_{1}}\left(q_{1}\right) n_{\lambda_{4}}\left(q_{4}\right)+n_{\lambda_{1}}^{2}\left(q_{1}\right)\right)}{\left[\omega^{2}-\tilde{\Omega}_{\lambda_{4}}^{2}\left(q_{4}\right)\right]}\right]\right\} \\
& G_{7}^{\lambda}\left(q_{4}, \omega\right)=\left\{\sum_{q:} \frac{3}{16}\left(\sum_{i j i} g_{i j \lambda} \alpha_{i}^{\dagger}\left(\mu q_{1}\right) \alpha_{j}\left(\mu q_{1}\right) b_{\dot{\mu}_{4}}^{\dagger}\left(q_{4}\right) b_{2 j_{4}}\left(q_{4}\right)\right)^{2} / \tilde{\omega}_{\mu}^{2}\left(q_{1}\right) \tilde{\Omega}_{\lambda_{4}}\left(q_{4}\right)\right.
\end{aligned}
$$

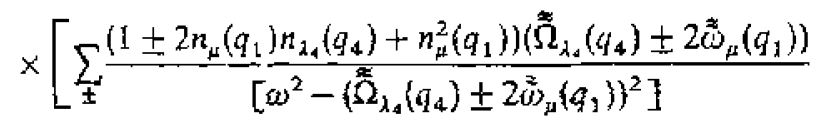

$$
\begin{aligned}
& \left.\left.+\frac{2 \Omega_{\lambda_{4}}\left(q_{4}\right)\left(1+2 n_{4}\left(q_{1}\right) n_{\lambda_{4}}\left(q_{4}\right)+n_{\mu}^{2}\left(q_{1}\right)\right)}{\left[\omega^{2}-\Omega_{i_{4}}^{z}\left(q_{4}\right)\right]}\right]\right\} \text {. }
\end{aligned}
$$

Using these expressions the value of the Green's function (14) can be written in a convenient form

where

$$
\operatorname{Lim}_{\xi \rightarrow 0} G(\omega+j \varepsilon)=G^{\prime}(\omega)-j G^{\prime \prime}(\omega)
$$

$$
G^{\prime}(\omega)=\Omega_{\lambda_{4}}\left(q_{4}\right)\left(\omega^{2}-\Omega_{\lambda_{4}}^{2}\left(q_{4}\right)\right) i \pi\left[\left\{\omega^{2}-\Omega_{\lambda_{4}}^{2}\left(q_{4}\right)\right\}^{2}+\Omega_{\lambda_{4}}^{2}\left(q_{4}\right) \Gamma_{\lambda_{4}}^{2}\left(q_{4}, \omega\right)\right]
$$

and

$$
G^{\prime \prime}(\omega)=\Omega_{\lambda_{4}}^{2}\left(q_{4}\right) \Gamma_{\lambda_{4}}\left(q_{4}, \omega\right) / \pi\left[\left\{\omega^{2}-\Omega_{\lambda_{4}}^{2}\left(q_{4}\right)\right\}^{2}+\Omega_{\lambda_{4}}^{2}\left(q_{4}\right) \Gamma_{\lambda_{4}}^{2}\left(q_{4}, \omega\right)\right],
$$

where $\Delta_{\lambda_{4}}\left(q_{4}, \omega\right)$ and $\Gamma_{\lambda_{4}}\left(q_{43} \omega\right)$ represent phonon frequency shift and width respectively and $\Omega_{1_{4}}\left(q_{4}\right)$ is the soft mode frequency which is given by

$$
\Omega_{\lambda_{4}}^{2}\left(q_{4}, \omega\right)=\bar{\Omega}_{\lambda_{4}}^{2}\left(q_{4}\right)+\Omega_{\lambda_{4}}\left(q_{4}\right) \Delta_{\lambda_{4}}\left(q_{4}, \omega\right) \text {. }
$$

The phonon frequency shift and width are given by

and

$$
\Omega_{\lambda_{4}}\left(q_{4}\right) \Delta_{\lambda_{4}}\left(q_{4}, \omega\right)=\sum_{i=1}^{7} G_{i}^{\prime \lambda}\left(q_{4}, \omega\right)
$$

$$
\Omega_{\lambda_{4}}\left(q_{4}\right) \Gamma_{i .4}\left(q_{4}, \omega\right)=\sum_{i=1}^{7} G_{i}^{\prime \prime \lambda}\left(q_{4}, \omega\right)
$$

where $G_{i}^{\prime \lambda}\left(q_{4}, \omega\right)$ and $G_{i}^{\mu \lambda}\left(q_{4}, \omega\right)$, the real and imaginary parts of $G_{i}^{\lambda}\left(q_{4}, \omega\right)$ are obtained by using the formula

$$
\lim _{x \rightarrow 0} \frac{1}{x \pm j c}=\frac{1}{x} \mp j \pi \delta(x) .
$$

The phonon frequencies and widths are obtained for different modes in different phases by using the parameter values given by Pytte (1972). Here, for example, the tetragonal phase has been taken. 


\section{Optical phonon frequencies and widths in tetragonal structure}

For the tetragonal distortion corresponding to a static displacement of ions along cube axis, only one of the components of the soft mode coordinates is different from zero and one sets

$$
\begin{aligned}
A_{\lambda} & =A \delta_{\lambda_{3}} \\
b_{\lambda \lambda^{\prime}} & =\delta_{\lambda \lambda^{\prime}},
\end{aligned}
$$

where 3 -axis has been chosen as the $c$-axis

$$
e_{i}=0 \text { for } i>3 \text { and } e_{1}=e_{2} \neq e_{3} \text {. }
$$

From (12) and the form of the interaction potential $v_{\lambda 2}(q)$, it follows that pure longitudinal and transverse modes are obtained for the propagation vector in 1,2 plane and along the 3-axis. For $\vec{q} \perp \bar{c}$ there are two different transverse modes $\tilde{\Omega}_{1 \mathrm{~T}}[E(\mathrm{TO})]$ and $\vec{\Omega}_{3 \mathrm{r}}\left[A_{1}\right.$ (TO) $]$ and one longitudinal mode $\tilde{\Omega}_{1 \mathrm{~L}}[E(\mathrm{LO})]$. For $\vec{q} \| \overrightarrow{\mathrm{c}}$ there is a doubly degenerate mode $\widetilde{\Omega}_{1 \mathrm{r}}[E(\mathrm{~T} 0)]$ and one longitudinal mode $\widehat{\Omega}_{3 \mathrm{~L}}\left[A_{1}(\mathrm{~L} 0)\right]$.

The free energy is given by

$$
F=\langle H\rangle-T S \text {, }
$$

where $\langle H\rangle$ is the average Hamiltonian and

$$
S=k_{B} \sum_{\lambda, q}\left[\left(1+n_{2}(q)\right) \ln \left(1+n_{\lambda}(q)\right)-n_{\lambda}(q) \ln n_{\lambda}(q)\right]
$$

is the entropy of the system and $n_{2}(q)$ the Bose occupation number. In the extremum condition the derivatives of free energy with respect to order parameter $A$ and strain coefficients are zero. Using this condition, the normal mode frequencies from (12) for tetragonal structute are obtained as

$$
\begin{aligned}
\widetilde{\Omega}_{\mathrm{iT}}^{2}= & \left(2 \Gamma_{2}-\Gamma_{1}\right) A^{2}+\left(2 \Gamma_{2}-3 \Gamma_{1}\right)\left(\Delta_{3}-\Delta_{1}\right)+2\left(e_{3}-e_{1}\right)\left(G_{12}-G_{i 1}\right) \\
& +\sum_{i j}\left(g_{i j 1}-g_{i j 3}\right)\left(e_{i} e_{j}+D_{i j}\right)
\end{aligned}
$$

with

$$
D_{i j}=\sum_{\mu q} \frac{\alpha_{i}(\mu q) \alpha_{j}(\mu q) \operatorname{coth}\left(\beta \omega_{j 2}(q) / 2\right)}{2 \omega_{\mu}(q)}
$$

and

$$
\tilde{\Omega}_{3 \mathrm{~T}}^{2}=2 \Gamma_{1} A^{2}, \text { with } \Delta_{\lambda}=\left(2 \tilde{\Omega}_{2}\right)^{-1} \operatorname{coth}\left(\beta \tilde{\Omega}_{\lambda} / 2\right), \quad \lambda=1,3 .
$$

It can be noted that $\widehat{\Omega}_{1 \mathrm{~T}}$ has a complicated temperature dependence whereas $\Omega_{3 \mathrm{~T}}$ is proportional to $A$ and thus the polarization, which is proportional to $\left(T-T_{c}\right)^{1 / 2}$ (Pytte 1972). Using parameters given above the phonon frequency shifts and widths for different modes can be calculated from (20) and (21) respectively.

\section{Acoustic phonon frequencies and widths}

The acoustic phonon frequency widths and shifts which lead to the expressions for acoustic attenuation, are obtained analogously from acoustic phonon Green's function

$$
G_{\mu}\left(t-t^{\prime}\right)=\left\langle\left\langle S_{\mu_{3}}\left(q_{3}\right)_{t}: S_{\mu_{4}}\left(q_{4}\right)_{t^{\prime}}\right\rangle,\right.
$$


which gives

where

$$
G_{\mu}(\omega)=\omega_{\mu}\left(q_{4}\right) \delta_{\mu_{3} \mu_{4}} \delta_{43 q_{4}} / \pi\left[\omega^{2}-\tilde{\omega}_{\mu_{4}}^{2}\left(q_{4}\right)-p_{\mu_{4}}\left(q_{4}, \omega\right)\right]
$$

$$
\begin{aligned}
\tilde{\omega}_{\mu_{4}}^{2}\left(a_{4}\right)= & \bar{\omega}_{\mu_{4}}\left(q_{4}\right)+\frac{1}{2} \sum_{i j \lambda \lambda_{1} q_{1}} g_{i j \lambda} \alpha_{i}^{t}\left(j q_{4}\right) \alpha,\left(\lambda q_{q_{4}}\right) b_{\lambda_{1}}^{\dagger}\left(q_{1}\right) b_{\lambda \lambda_{1}}\left(q_{1}\right) \\
& \times\left[\frac{\operatorname{coth}\left(\beta \tilde{\Omega}_{\lambda_{1}}\left(q_{1}\right) / 2\right)}{\bar{\Omega}_{\lambda_{1}}\left(q_{1}\right)}\right] \\
\tilde{\omega}_{\mu_{1}}\left(q_{4}\right)= & \omega_{0}^{2}\left(\mu_{4} q_{4}\right)+\sum_{i j k} g_{i j \lambda} A_{\lambda}^{2} \alpha_{t}\left(\mu_{4} q_{4}\right) \alpha_{j}\left(\mu_{4} q_{4}\right)
\end{aligned}
$$

and

$$
p_{\mu_{+}}\left(q_{4}, \omega\right)=\sum_{i=1}^{4} G_{i}^{\mu}\left(q_{4}, \omega\right)
$$

with

$$
\begin{aligned}
& G_{1}^{\mu}\left(q_{4}, \omega\right)=\left\{4\left(\sum_{i, \lambda^{\prime}} G_{i \lambda \lambda^{\prime}} A_{\lambda^{\prime}}+\sum_{i j \lambda} g_{i j \lambda} A_{\lambda} e_{j}\right)^{2}\left|\alpha_{i}\left(\mu q_{4}\right) b_{\lambda \lambda}\left(q_{4}\right)\right|^{2} /\right. \\
& \left.\left[\omega^{2}-\overline{\mathbf{\Omega}}_{\lambda_{4}}^{2}\left(q_{4}\right)-p_{\lambda_{4}}\left(q_{4}, \omega\right)\right]\right\} \\
& G_{2}^{\mathrm{E}}\left(a_{4}, \omega\right)=\left\{\sum _ { \boldsymbol { q } _ { 1 } } \left(\sum_{\lambda, \lambda^{*}} G_{i \lambda \lambda^{\prime}, \alpha_{i}}\left(\mu q_{4}\right) b_{i \lambda_{1}}^{\dagger}\left(q_{1}\right) b_{\lambda^{\prime} \lambda_{1}}\left(q_{1}\right)\right.\right. \\
& \left.\left.+\sum_{i j \lambda} g_{i j \lambda} E_{j} a_{i}\left(\mu q_{4}\right) b_{\mu_{1}}^{\dagger}\left(q_{1}\right) b_{\lambda \lambda_{1}}\left(q_{1}\right)\right)^{2} \frac{n_{\lambda_{1}}\left(q_{1}\right)}{2 \Omega_{\lambda_{1}}\left(q_{1}\right)\left[\omega^{2}-4 \Omega_{\lambda_{1}}^{2}\left(q_{1}\right)\right]}\right\} \\
& G_{3}^{\mu}\left(q_{4}, \omega\right)=\left\{\sum_{q_{1}}\left(\sum_{i j \lambda} g_{i j \lambda} A_{\lambda} b_{\lambda \lambda_{1}}\left(q_{1}\right) \alpha_{i}\left(\mu q_{4}\right) \alpha_{j}\left(\mu q_{4}\right)\right)^{2} \frac{1}{\omega_{\mu}\left(q_{4}\right) \bar{\Omega}_{\lambda_{1}}\left(q_{1}\right)}\right. \\
& \left.\times\left[\sum_{ \pm} \frac{\left(n_{\lambda_{1}}\left(q_{1}\right) \pm n_{\mu}\left(q_{4}\right)\right)\left(\tilde{\omega}_{\mu}\left(q_{4}\right) \pm \tilde{\Omega}_{\lambda_{1}}\left(q_{1}\right)\right)}{\left[\omega^{2}-\left(\tilde{\omega}_{p}\left(q_{4}\right) \pm \bar{\Omega}_{\lambda_{1}}\left(q_{1}\right)\right)^{2}\right]}\right]\right\}
\end{aligned}
$$

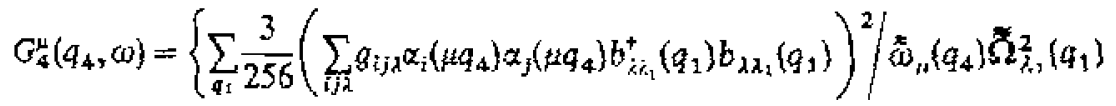

$$
\begin{aligned}
& \times\left[\sum_{ \pm} \frac{\left(1 \pm 2 n_{\lambda_{1}}\left(q_{1}\right) n_{\mu}\left(q_{4}\right)+n_{\lambda_{1}}^{2}\left(q_{1}\right)\right)\left(\tilde{\omega}_{\mu}\left(q_{s}\right) \pm 2 \bar{\Omega}_{\lambda_{1}}\left(q_{1}\right)\right)}{\left[\omega^{2}-\left(\omega_{\mu}\left(q_{4}\right) \pm 2 \hat{\Omega}_{\lambda_{1}}\left(q_{1}\right)\right)^{2}\right]}\right. \\
& \left.\left.+\frac{2 \hat{\omega}_{\mu}\left(q_{4}\right)\left(1+2 n_{\mu}\left(q_{4}\right) n_{\lambda_{1}}\left(q_{1}\right)+n_{\lambda_{1}}^{2}\left(q_{1}\right)\right)}{\left[\omega^{2}-\bar{\omega}_{\mu}^{2}\left(q_{4}\right)\right]}\right]\right\} \text {. }
\end{aligned}
$$

where $n_{k}$, the phonon accupation number

and

$$
n_{k}=\frac{\omega_{k}}{\tilde{\omega}_{k}} \operatorname{coth}\left(\beta \tilde{\omega}_{k / 2}^{\star}\right)
$$

$$
\beta=\left(k_{B} T\right)^{-1},
$$

$k_{B}$ being the Boltzmann constant and $T$ the absolute temperature.

The real and imaginary parts of $p_{u},\left(q_{4}, \omega\right)$ are obtained by using (22) and the 
acoustic phonon widths are obtained as

$$
\Gamma_{\mu}\left(q_{4}, \omega\right)=\frac{\pi}{\omega_{\mu}\left(q_{4}\right)} \operatorname{Im} p_{\mu}\left(q_{4}, \omega\right)
$$

The expression for acoustic attenuation constant is given by

$$
\alpha_{\mu}(q, \omega)=\Gamma_{\mu}(q, \omega) / C_{\mu}(q)
$$

where $C_{n}(q)$ is the velocity of acoustic wave, $\mu=L$ for longitudinal and $T$ for transverse wave.

\section{Comparison with experiments and discussion}

\subsection{Dielectric constant and iangent loss}

Following Kubo (1957) and Zubarev (1960) the real part of dielectric constant is given by

$$
k^{\prime}(\omega)-1=-8 \pi^{2} N \underline{\mu}^{2} G^{\prime}(\omega)
$$

where $\mu$ is the effective dipole moment per unit cell and $N$ is the number of cells in the sample.

The dielectric loss (tan $\delta$ ) is defined as the ratio of imaginary and real parts of dielectric constant and can be written as

$$
\tan \delta=G^{\prime \prime}(\omega) / G^{\prime}(\omega)
$$

Thus the retarded one-phonon Green's function is enoug! to determine the dielecric constant and loss tangent. Using (32) and (18a), the real part of the dielectric constant can be written as

$$
k^{\prime}(\omega)-1=-8 \pi N \underline{\mu}^{2} \Omega_{\lambda}(q)\left(\omega^{2}-\Omega_{3}^{2}(q)\right) /\left[\left(\omega^{2}-\Omega_{\lambda}^{2}(q)\right)^{2}+\Omega_{\lambda}^{2}(q) \Gamma_{\lambda}^{2}(q, \omega)\right]
$$

For the perovskite crystals the microwave or lower frequency is much smailer than the optical soft mode frequency $\Omega_{\text {sof }}\left(\omega / \Omega \simeq 10^{-3}\right)$ and no relaxation efiects are observed. Due to this appreciable difference between $\omega$ and the optical soft mode frequency, (34) can be written as

$$
k^{\prime}(\omega)=8 \pi N \mu^{2} \Omega_{\lambda}(q) / \Omega_{\lambda}^{2}(q), \quad \text { as } k^{\prime}(\omega) \gg 1 .
$$

In the vicinity of the transition temperature (25b) with the shift term gives $\Omega_{\text {sor }} \propto\left(T-T_{c}\right)^{1 / 2}$, and (35) becomes

$$
k^{\prime}(\omega)=\text { constant } /\left(T-T_{c}\right)
$$

This is the Curie-Weiss law.

The tangent loss is given by

$$
\tan \delta=\Gamma_{\lambda}(q, \omega) /\left[\omega^{2}-\Omega_{\lambda}^{2}(q)\right],
$$

where $\Gamma_{\lambda}(q, \omega)$ is given by (21). For $\omega \ll \Omega_{\lambda}(q)$, (37) becomes

$$
\tan \delta=-\Gamma_{i}(q, \omega) / \Omega_{\lambda}^{2}(q, \omega)=\omega\left(\alpha+\beta T+\gamma T^{2}\right) /\left(T-T_{c}\right)
$$


where $\alpha$ is harmonic and defect contribution, $\beta$ and $\gamma$ are due to three and four phonon anharmonic interaction terms of the lattice.

Thus a possible explanation of the origin of temperature dependence of microwave anci lower frequency loss tangent and dielectric constant is possible if the paraelectricity of $\mathrm{ABO}_{3}$ perovskites is regarded as originating from the temperature dependent optical soft phonon frequency. A transverse radiation field derives the solt transverse optical mode of the material on the forced vibration. Energy is transferred from the electromagnetic field to this lattice node and is degraded into other vibrational modes. Equations (36) and (38) describe the behaviour of $\mathrm{SrTiO}_{3}$ and $\mathrm{BaTiO}_{3}$ (Rupprecht and Bell 1962; Benedict and Durand 1958) quite well. Paletto et al (1974) measured the variation of permittivity and $\tan \delta$ of $\mathrm{BaTiO}_{3}$ with temperature. The anomaly near the transition temperature can be explained by the fact that the optical mode frequency softens near the transition temperature giving a large value of dielectric constagt and loss near the transition points (36) and (38).

Similarly, the phonon frequency and damping constant in the first approximation comes out to be

and

$$
\Omega_{\lambda}^{2}(q)=\alpha+\beta T+\gamma T^{2}
$$

$$
\Gamma_{\lambda}(q)=\alpha^{\prime}+\beta^{\prime} T+\gamma^{\prime} T^{2}
$$

which on fitting with the experimental results obtained by others for $\mathrm{BaTiO}_{3}$ (Lupsin et al 1980), $\mathrm{SrTiO}_{3}$ and $\mathrm{LaAlO}_{3}$ (Feder and Pytte 1970) give the values of $\alpha, \beta$ and $\gamma$ 's for different modes in different phases as listed in tables 1 and 2 .

The Curie-Weiss behaviour of tangent loss in $\mathrm{SrTiO}_{3}$ and $\mathrm{SrTiO}_{3}$ doped with impurity (Rupprecht and Bell 1962) shows that this contribution is due to the temperature-independent term $\alpha$ in (38). This suggests that imperfections cause

\begin{tabular}{|c|c|c|c|c|c|}
\hline & \multicolumn{2}{|c|}{$\mathrm{BaTiO}_{3}$} & \multicolumn{3}{|c|}{$\mathrm{SrTtO}_{3}$} \\
\hline & \multirow{2}{*}{$\frac{\text { Cubic phase }}{F_{1 \text { d (soft mode) }}}$} & \multirow{2}{*}{$\frac{\text { Tetragonal }}{E(T O)}$} & \multirow{2}{*}{$\frac{\text { Cubic }}{F_{14}}$} & \multicolumn{2}{|c|}{ Tetragonal } \\
\hline & & & & $E(T 0)$ & $A_{1}(\mathrm{TO})$ \\
\hline $\begin{array}{l}8 \mathrm{~cm}^{-1} \\
8 \mathrm{~cm}^{-1} \mathrm{~K}^{-1} \\
y \mathrm{~cm}^{-1} \mathrm{~K}^{-2}\end{array}$ & $\begin{array}{l}-63.2 \\
0.2865 \\
-8.4 \times 10^{-5}\end{array}$ & $\begin{array}{l}-76.48 \\
0.57 \\
-6.12 \times 10^{-4}\end{array}$ & $\begin{array}{l}-53.59 \\
0.737 \\
-1.49 \times 10^{-3}\end{array}$ & $\begin{array}{l}17.59 \\
-0.0915 \\
3.26 \times 10^{-4}\end{array}$ & $\begin{array}{l}4479 \\
\quad 0-048 \\
-25 \times 10^{-3}\end{array}$ \\
\hline
\end{tabular}

Table 1. Values of $\alpha, \beta$ and $\gamma$ obtained by fitting (39) with experimental results (figures $1,2,3$ ).

\begin{tabular}{|c|c|c|c|}
\hline & \multicolumn{2}{|c|}{$\mathrm{IAAIO}_{3}$} & \\
\hline & Cubie & Trigona! & \\
\hline & $F_{\text {[u }}$ & $\mathrm{E}(\mathrm{TO})$ & $A_{1}(\mathrm{TO})$ \\
\hline $\begin{array}{l}x \mathrm{~cm}^{-1} \\
\beta \mathrm{cm}^{-1} \mathrm{~K}^{-1} \\
\gamma \mathrm{cm}^{-1} \mathrm{~K}^{-2}\end{array}$ & $\begin{array}{l}-101.82 \\
0.2096 \\
-6.31 \times 10^{-5}\end{array}$ & $\begin{array}{l}128.05 \\
0.1534 \\
1.87 \times 10^{-4}\end{array}$ & $\begin{array}{l}37.03 \\
-0.009 \\
-4.1 \times 10^{-5}\end{array}$ \\
\hline
\end{tabular}

Tabie 1. (Continued) 
damping. The imperfections couple the soft mode to other modes and provide a mechanism for scattering energy out of the driven mode. At higher temperatures the loss deviates strongly from the Curie-Weiss type behaviour and increases linearly with temperature. This assumes that at higher temperature lattice, anharmonicity is responsible for the observed loss. This discussion also applies ${ }^{\circ} \mathrm{BaTiO}_{3}$ in the

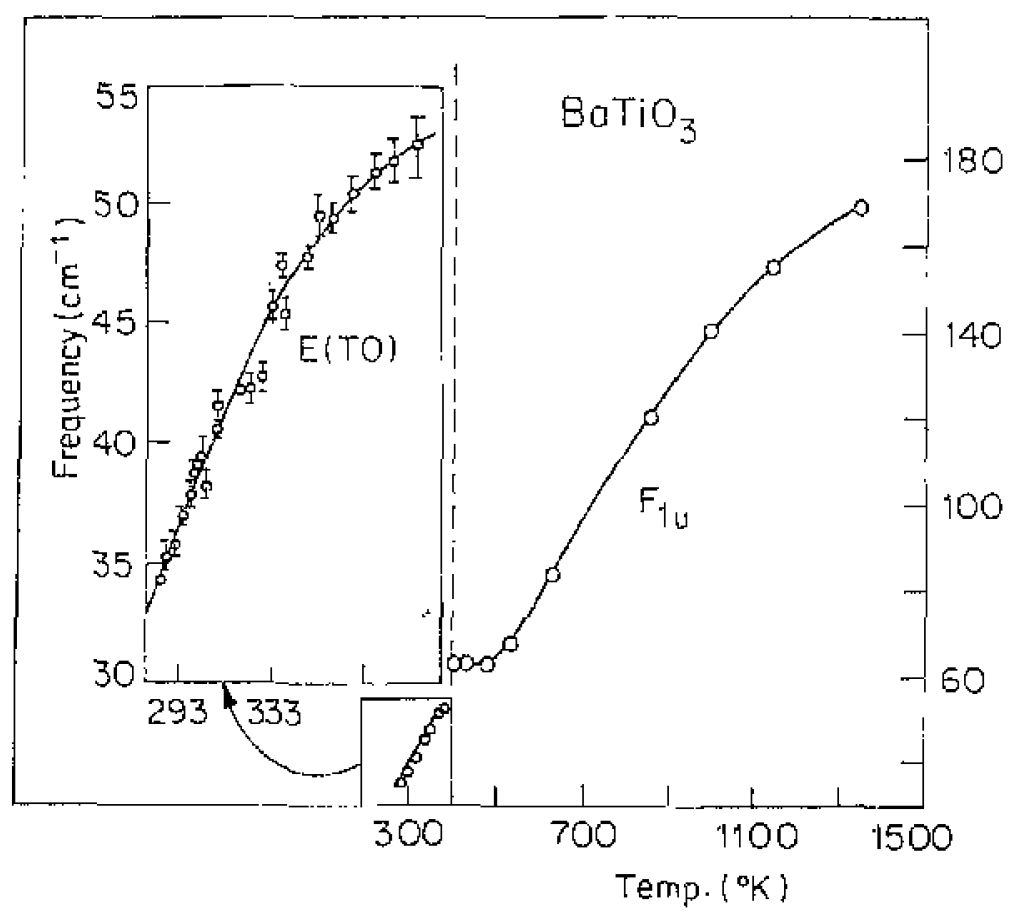

Figure 1. Temperature dependence of $E(T 0)$ and $F_{1 \mu}$ modes for BaTiO ${ }_{3}$. $\phi$ and $O$ points represen! experimental results and the solid lines represent (39) with $\alpha, \beta$, $y$ from table 1 .

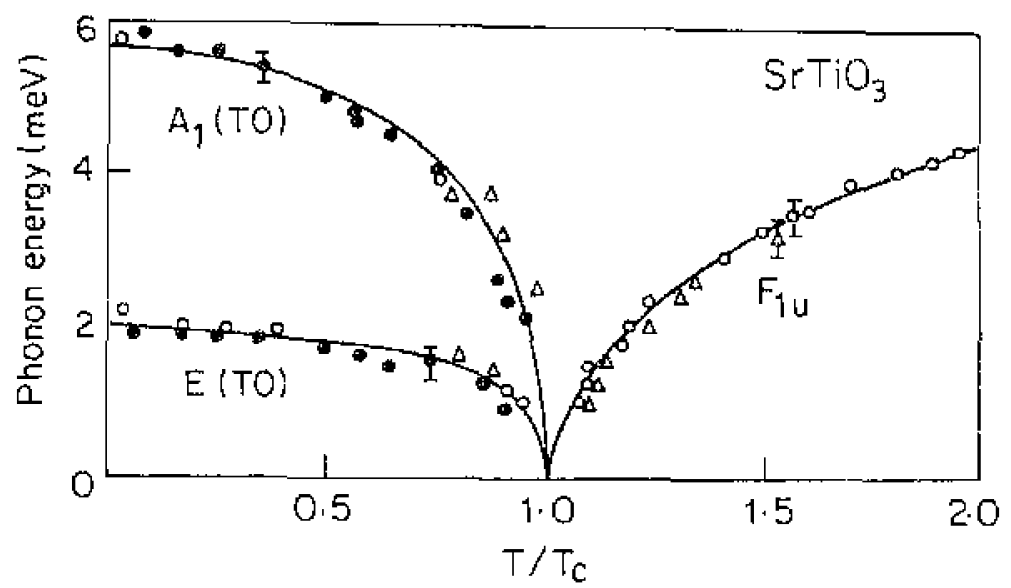

Figure 2. Tenuperature dependence of optical phonon frequency for $\mathrm{SrTiO}_{3}$. The points on and $\Delta$ are experimental restits $(O$, Fleury et $a l$; , Shitane and Yamada; $\triangle$, Cowlcy at af) and the solid curves represent (39) with $\alpha, \beta, \gamma$ from table 1. 


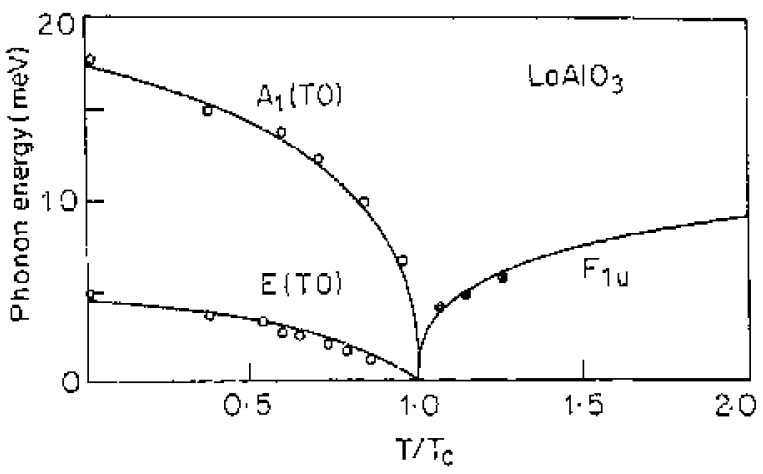

Figure 3. Temperature dependence of optical phonon frequencies for $\mathrm{LaAlO}_{3}, Q$ and cepresent experimental results ( $\bullet$, Axe et al; 0 , Scott) and solid curves represent (39) with $z, \beta, y$ from table 1 .

Talth 2. Values of $\alpha^{\prime}, \beta^{\prime}, \gamma^{\prime}$ obtained by fitting (40) with experimental results, figures 4,5 , for $\mathrm{BaTiO}_{3}$.

\begin{tabular}{lccc} 
& $F_{1 \mathrm{u}}$ (soft mode) & $A_{1}(\mathrm{TO})$ & $E(\mathrm{TO})$ \\
\hline$\alpha \mathrm{cm}^{-1}$ & 13.475 & 1959 & 98.84 \\
$\beta^{\prime} \mathrm{cm}^{-1} \mathrm{~K}^{-1}$ & 0.31 & -11.784 & -0.34 \\
$\gamma^{\prime} \mathrm{cm}^{-1} \mathrm{~K}^{-2}$ & $-9.75 \times 10^{-5}$ & 0.019 & $1.25 \times 10^{-3}$ \\
\hline
\end{tabular}

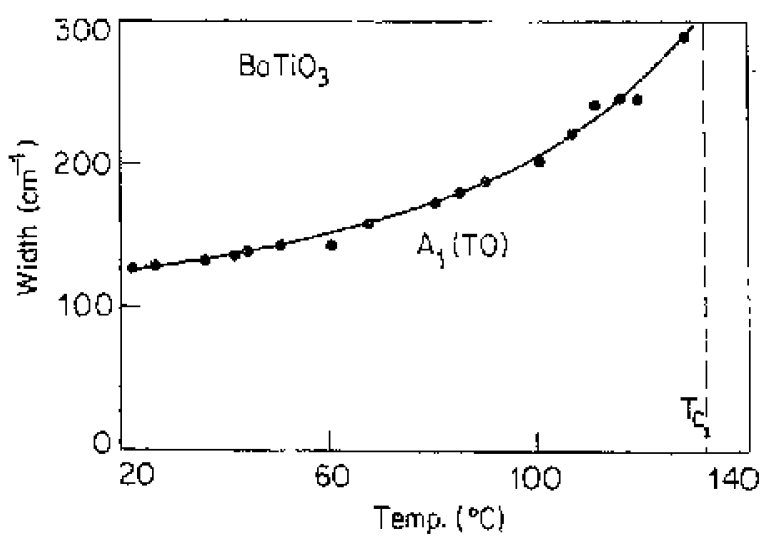

Figure 4. Temperature dependence of width of $A_{1}(\mathrm{TO})$ mode in $\mathrm{BaTiO}_{3}$. - show experimental results and the solid curve is represented by (40) with $\alpha^{\prime}, \beta, \gamma^{\prime}$ from table 2.

paraelectric phase. The observed loss tangent of $\mathrm{BaTiO}_{3}$, however, is some orders of magnitude larger than the $\mathrm{SrTiO}_{3}$ value (Benedict and Durand 1958; Stern and Luric 1961). The temperature dependence of loss tangent is a reflection of temperature dependence of frequency of the transverse polarization mode-the optical soft mode. The high loss of polycrystalline samples of $(\mathrm{Ba}-\mathrm{Sr}) \mathrm{TiO}_{3}$ mixture and single crystalline samples of $\mathrm{BaTiO}_{3}$ (Stern and Luric 1961) exhibit the loss tangent represented by 


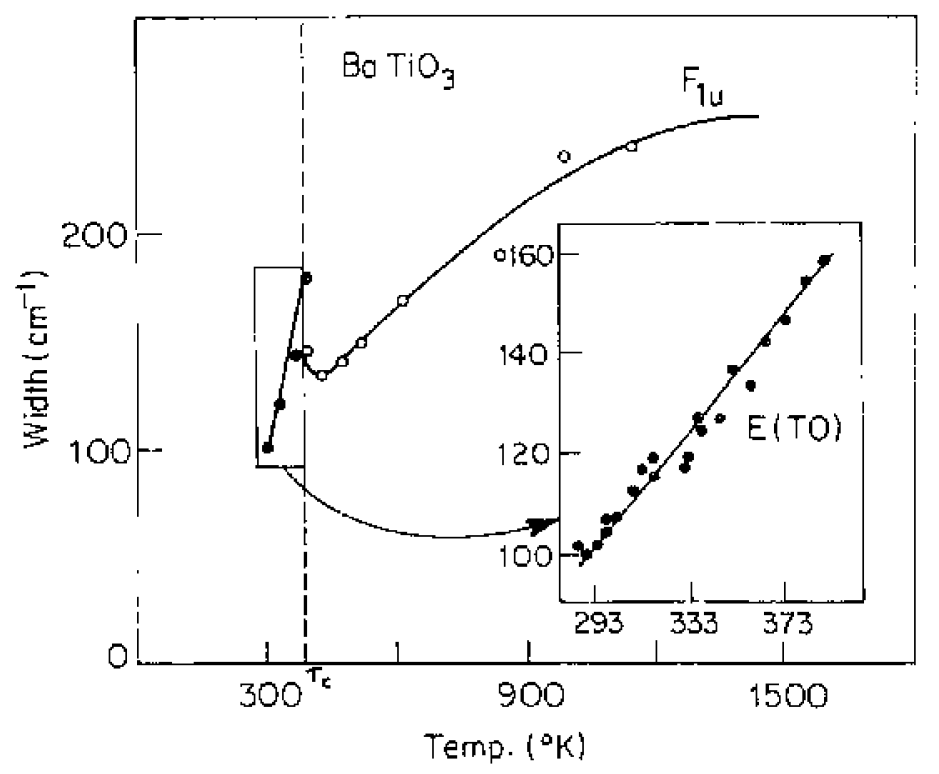

Figure 5. Temperature dependence or damping of $F_{14}$ and $E(\mathrm{TO})$ modes in $\mathrm{BaTiO}_{3} .0$ and - represent experimental results and solid curves are represented by (40) with $\alpha^{\prime}, \beta^{\prime}, y^{\prime}$ lrom table 2 .

(38). Other perowskites, e.g., $\mathrm{CaTiO}_{3}$ (Linz and Harrington 1958), $\mathrm{KTaO}_{3}, \mathrm{KTaO}_{3}$ : $\mathrm{NaTaO}_{3}$ (Agrawal and Rao 1970) also exhibit the same behaviour.

The frequency dependence of loss tangent for perovskites is linear and so is the temperature dependence at higher temperatures. The resultant increase in loss is not due to the bulk electronic semiconduction because this would in turn lead to a reciprocal frequency dependence of loss tangent. The temperature dependence of loss does not appear to be exponential. Thus, third and fourth order anharmonicity may be responsible for the observed behaviour of loss tangent.

\subsection{Acoustic attenuation}

Expression for acoustic attenuation coefficient is given by (31). In the vicinity of the transition temperature $\Omega_{\mathrm{k} \alpha \mathrm{f}} \propto\left(T-T_{\mathrm{c}}\right)^{1 / 2}$ and the temperature dependence of damping constant of the acoustic wave near the transition temperature, can be expressed as

$$
\begin{aligned}
\Gamma_{\mu}(q, \omega)= & {\left[A_{1}(\omega)+\left\{A_{2}(\omega)+\frac{A_{3}(\omega)}{\left(T-T_{c}\right)^{1 / 2}}+\frac{A_{4}(\omega)}{\left(T-T_{c}\right)^{3 / 2}}\right\} T\right.} \\
& \left.+\left\{A_{5}(\omega)+\frac{A_{6}(\omega)}{\left(T-T_{c}\right)^{3 / 2}}+\frac{A_{7}(\omega)}{\left(T-T_{c}\right)^{2}}\right\} T^{2}\right]
\end{aligned}
$$

where $A_{t}(\omega)$ are temperature independent terms.

Acoustic wave velocity decreases anomalously (Naithani and Semwal 1980) in the vicinity of the transition temperature. This also contributes to the anomalous increase of the attenuation constant near transition temperature of the displacive ferroelectrics. These results agree with the results of Tani and Tsuda (1969). At the temperature 
which is away from the Curie tempetature, the soft mode frequency is nearly temperature-independent and so is the acoustic wave velocity. Thus, the acoustic attenuation constant can be written as

$$
\Gamma_{\mu}(q, \omega)=A_{1}^{\prime}(q, \omega)+A_{2}^{\prime}(q, \omega) T+A_{3}(q, \omega) T^{2} .
$$

This type of behaviour has been observed for $\mathrm{LiNbO}_{3}$ (Lemanov et al 1969), BaTiO, (Hueter and Neuhaus 1955) and $\mathrm{SrTiO}_{3}$ (Nava et al 1969) experimentally which confirms that higher order anharmonicity dominates at higher temperatures. Thus the experimental and theoretical results reveal that the binding forces between atons do not show the ideal spring behaviour and consequently the restoring forces are proportional to higher powers of displacement as well.

\section{References}

Agrawal M D and Rao K Y 1970 J. Phys. C3 1120

Anderson P W 1960 Froc. Conf, on Physics of Dielectrics (ed.) G I Skanavi (Moscow: Academy of Sciences) p. 290

Benedict T S and Durand J L 1958 Phys. Rev. 1091091

Cochran W 1959 Phys. Rev. Lett. 3412

Cochran W and Zia A 1968 Phys. Sittus Solidi 25273

Cowley R A, Buyers W J L and Dolling G 1969 Solid State Commun. 7181

Feder J and Pytte E 1970 Phys. Rev. Bl 4803

Gairola R P and Semwal B S 1977 J. Phys. Soc. Jpn 42975

Hueter T F and Neuhaus D P 1955 J. Acoust. Soc. Am. 27292

Kobayashi K K 1968 J. Phys. Sac. Jpn. 24497

Kubo R 1957 J. Plitsis Soc. Jpn 12570

Lemanov $V \vee$, Smolenskii G A and Sherman A B 1969 Sov. Phys. Solid State 11524

Litz A and Harrington K $1958 \mathrm{~J}$. Chem. Phys. 28824

Lupsir Y, Servoin J L and Gervais F 1980 J. Phys. C13 3761

Naithani U C and Semwal B S 1980 Pramana - J. Phys. 1419

Nava R, Cellarotti R, Ceva $\mathrm{H}$ and Martiret A 1969 Phys. Rev. 1881456

Paletto J, Grange G. Goutte R and Eyraud L 1974 J. Phys. D7 78

Pytte E 1972 Phys. Rev. BS 3758

Rupprecht $\mathrm{G}$ and Bell R O 1962 Phys, Rev. 1251915

Semwal B S 1972 Studies of latice anharmonicity and isotopic imperfection in crystals, D.Phil. thesis, Allahabad University, Allahabad

Semwal B S and Sharma P K 1972 Phys. Rev. B5 3909

Semwal B S and Sharma P K 1974 J. Math. Phys. 15648

Sernwal B S and Sharma P K 1974 Progr. Theor. Phys. Japan 51639

Stern $E$ and Luric A 1961 Phys. Rev. 123117

Tani K and Tsuda N 1969 J. Phys. Soc, Japan 2693

Tokunaga M and Malsubara T 1966 Progr. Theor. Phys. 35981

Zubarev D N 1960 Phys. Usp. 3320 